\title{
Chronobiology, the Future of Medicine
}

\author{
Heon-Jeong Lee \\ Editor-in-Chief \\ Department of Psychiatry, Korea University College of Medicine, Seoul, Korea \\ Chronobiology Institute, Korea University, Seoul, Korea
}

Chronobiology, the study of biological rhythms, has made a significant contribution to the development of medicine in recent years. It is now clear that the circadian clock not only affects the body, but also has a significant impact on the mind and behavior. The discovery of the molecular basis of the circadian rhythm by Jeffrey Hall, Michael Rosbash, and Michael Young was a seminal contribution to the field of medicine and was recognized by the Nobel Prize in Physiology or Medicine, 2017 [1]. Chronobiology finds itself at the epicenter of future medical advances. In the past, clinical medicine did not pay much attention to the circadian rhythm as related to the human body and mind. Diagnoses were made based on the physical examination, laboratory tests, or imaging tests at cross-sectional time points. There was an underlying assumption that the test performed at a certain time did not yield results different from the test conducted at other times. However, it soon became apparent that the test results may vary depending on the time of day. In addition, it was also seen that disruption or misalignment of the circadian rhythm may play an important role in the pathogenesis and treatment of diseases.

Circadian clock is an internal timekeeping mechanism that controls most of the body's functions. The suprachiasmatic nucleus is the master regulator of biological clock that regulates the function of various organs such as brain, liver, kidney, and heart, to name a few. Disruption of circadian clock either due to shift work, jet lag, or severely delayed sleep phase significantly increases the risk of developing various diseases such as metabolic disorders, cardiovascular diseases, cancer, neurodegenerative disorders, and mental disorders [2-4]. This suggests that circadian clock-controlled actions play essential roles in human physiology and behavior. The liver as an independent circadian oscillator, possibly entrained by food timing. There is a vast need for additional research in this area, especially as it impacts shift work. Since disruption in the biological clock is associated with a variety of human diseases, restoring the circadian clock can help with the treatment or prevention of several diseases. Restoration of circadian clock has been shown to improve the clinical symptoms of oxidative stress and inflammation [5].

Over the past several decades, the development of circadian rhythm monitoring has been limited in part due to a lack of objective tools for continuous, simple, non-invasive quantification. The development of wearable sensor devices and mathematical models for the processing of big data will aid in accurately quantifying circadian disruption. Such techniques are important in precision medicine to be able to detect healthy lifestyles and diagnose and treat a variety of diseases. Wearable devices involve an attachment or a sensor placed directly on the body (e.g., wristband), or are attached to or embedded in the clothing. These devices directly track body movements or biometric information. A continuous biometric screening can help to track the health effects of fluctuations in the circadian rhythm. Strategies to restore circadian rhythm using the wearable devices can lead to new therapeutic interventions. Thus, measurement of the regulatory factors such as light, darkness, melatonin, and meal time can be used in conjunction with the rapid development of information and communication technology to treat many human diseases. The journal Chronobiology in Medicine was launched to emphasize the importance of chronobiology in the medical field. This new journal promises to become the primary platform for researchers to publish studies on all aspects of chronobiology. The aim of Chronobiology in Medicine is to be the premier venue to publish high quality research in the field of chronobiology, to lead the way to exciting discoveries in chronobiology, and to shape the future of medicine.

\section{REFERENCES}

1. Information on the 2017 Nobel prize in physiology or medicine. Available at: https://www.nobelprize.org/prizes/medicine/2017/press-release/. Accessed February 27, 2019.

2. Kervezee L, Kosmadopoulos A, Boivin DB. Metabolic and cardiovascular consequences of shift work: the role of circadian disruption and sleep disturbances. Eur J Neurosci 2018 Oct 25 [Epub]. Available at: https://doi.org/ 10.1111/ejn.14216.

3. Wyse CA, Celis Morales CA, Graham N, Fan Y, Ward J, Curtis AM, et al. Adverse metabolic and mental health outcomes associated with shiftwork in a population-based study of 277,168 workers in UK biobank. Ann Med 
2017;49:411-420.

4. Moon JH, Cho CH, Son GH, Geum D, Chung S, Kim H, et al. Advanced circadian phase in mania and delayed circadian phase in mixed mania and depression returned to normal after treatment of bipolar disorder. EBio-
Medicine 2016;11:285-295.

5. Wilking M, Ndiaye M, Mukhtar H, Ahmad N. Circadian rhythm connections to oxidative stress: implications for human health. Antioxid Redox Signal 2013;19:192-208 